\title{
1. Producing powerful numbers
}

\section{Frances Morphy, Will Sanders and John Taylor}

Census statistics are powerful numbers. Governments frequently use them in the allocation of important resources, such as seats in parliament or shares of expenditure between jurisdictional areas. More indirectly, they can be used to characterise social and economic situations among groups of people, and through that to drive important public policy debates. Who gets what, when and how from governments is often informed-if not determined-by what census statistics reveal about existing and projected numbers of people and their socioeconomic characteristics.

As researchers studying the socioeconomic circumstances of Indigenous Australians and contributing to Indigenous affairs policy debates, we have relied heavily on Australian census statistics in the past. In doing so, however, we have often had cause to wonder about the processes through which these statistics have been produced and the adequacy, accuracy and appropriateness of some of them-particularly in relation to Indigenous people in remote Australia. If these statistics are not adequately capturing the numbers or socioeconomic characteristics of Indigenous people in these areas, what effect might this be having on policy debates and on the allocation of public resources?

The Australian Bureau of Statistics (ABS) devised an Indigenous Enumeration Strategy (IES) because it had cause to be concerned itself about these questions with respect to the Indigenous population of Australia. The IES has evolved through the past 35 years to become a highly complex, multi-staged, multi-sited and multifaceted organisational exercise that consumes varying degrees of ABS resources and personnel continually between censuses, though obviously with most effort expended around the pivotal process of the enumeration itself. Over time, the ABS has committed steadily expanding resources to these collective exercises. Since 1971, when special enumeration procedures were first introduced in the Northern Territory and Western Australia, the IES has gradually become a truly national strategy, while the tasks and the personnel required to feed into IES processes have multiplied (Taylor 2002). In 2001, the direct cost of enumerating remote-area Indigenous populations was about \$2 million (or \$26 per capita) compared with the direct costs of about $\$ 49$ million (or $\$ 2.60$ per capita) for the total population. In 2006, the equivalent figures were $\$ 2.5$ million (or $\$ 35$ per capita) for the IES compared with $\$ 63$ million (about $\$ 3$ per capita) for the census overall.

This book provides, for the first time, an independent view of all stages of the enumeration process in remote, discrete Indigenous communities-from the 
training of the field staff and pre-census preparation through to data processing. The chapters are ordered in sequence to reflect the building blocks of what eventually emerges as population and housing data in census output tables. In this introductory chapter, we set the scene for the 2006 enumeration and the research project and, in the concluding chapter, we draw on our observations of the 2006 enumeration to make some quite radical suggestions for change in 2011 .

\section{The 2006 research project}

\section{Background}

In 2001 a team of CAEPR researchers observed the conduct of the census in three remote Aboriginal communities (Martin et al. 2002). While we were supportive of the interview and time extension adaptations of the 2001 IES, we observed that the two-form structure of a household form and separate individual forms then in use was very cumbersome and made large, somewhat unnecessary administrative demands on the local recruited census field staff - the Community Coordinators (CCs) and the collector-interviewers (CIs). In two of our observed 2001 locations - one in the Northern Territory and one in Queensland - the two-form structure was made to work largely by keeping household forms in the background and exposing interviewees only to the personal forms. In the third location observed in 2001, 12 days of very slow progress led to abandonment of the personal forms and a salvage operation that focused simply on the household forms. The two-form structure was just far too demanding on the interest and persistence of the interviewers, and their interviewees. CIs were becoming burnt out and falling by the wayside in the process of enumerating just a limited number of households.

Frances Morphy's work in 2001 (Morphy 2002) focused in addition on the highly inadequate construction of Indigenous households through particular census questions. She judged that the attempt to translate Indigenous kinship systems into Western terminology had been largely unsuccessful and that many of the Indigenous household descriptions in the census data were, as a consequence, of limited value. Morphy also focused on the 'legendary mobility' of Indigenous people in her observation area in north-east Arnhem Land and how, together with time extension of the collection process, this meant that a 'complete enumeration' was almost impossible. Double-counting and under-enumeration, she reasoned, were highly likely, but the extent of each would be difficult to assess.

An awareness of these Indigenous mobility issues had led the Northern Territory administration of the ABS, over some years, to attempt to count 'usual residents' of Indigenous communities, rather than following the standard census procedure of counting people present, with some provision for adding absent usual residents 
who might not be counted elsewhere. Morphy sympathetically considered the merits of counting usual residents, but ultimately argued against it on the grounds that a robust definition of 'usual resident' was hard to develop. Will Sanders' work on the Alice Springs town camps argued against the usual residents approach because it allowed people present - labelled 'visitors' — to slip through the enumeration process.

One final common theme in our 2001 observations was the limited social relevance to the circumstances of traditionally oriented Aboriginal people in remote Australia of many census questions and their pre-specified categorical answers - whether this was the difficulty of defining a dwelling and its associated household, of categorising an education level or course undertaken, or a question about religion or marriage, employment or looking for a job. Often this limited social relevance of questions and categorical answers would introduce humour to the collection process - for interviewers and interviewees. Lack of social relevance could, however, also lead to disinterest and disengagement.

On the basis of all these observations, in the final chapter of our monograph on the 2001 Census (Martin et al. 2002), we argued for essentially three reforms to the IES as we observed it operating in remote Australia. In opposition to some half-hearted moves in the Northern Territory towards enumerating usual residents, our first suggestion was to argue for a return to the general ABS approach of counting people present, with some facility for adding absent usual residents who might not be counted elsewhere. Our second suggestion was to argue for the reintegration of the special Indigenous personal and household forms into a single Indigenous household form designed to be administered by interview. Our third suggestion was for this form to be tailored more precisely to the circumstances of Indigenous people in remote Australia by the restriction of some questions asked and the development of more appropriate categorical answers.

The ABS formed an IES Working Group after the 2001 Census to consider ways of improving field design and methods. Sanders and Morphy were invited to be members of this group. It produced two key initiatives - one conceptual, the other practical - which corresponded with our first two ideas for reform. First, in 2006 there was to be a clear move back to the standard of enumerating people present in a dwelling at the time of the count, plus absent usual residents judged unlikely to be counted elsewhere. ${ }^{l}$ Second, the previous multi-form schedule of census questions was to be integrated into a single matrix-style Interviewer Household Form (IHF). Our third idea for reform, however - attempting to restrict this new form to a lesser number of questions of greatest social relevance to the circumstances of Indigenous people in remote areas - proved almost impossible

1 This standard approach produces what is sometimes referred to as a 'de facto' population. When people are placed back in their usual place of residence, the term 'de jure' population is sometimes used. 
for the ABS to implement. While individual questions and categorical responses to them could be modified slightly, the idea of leaving out any questions always met resistance. Any question left out would become a statistic for which there would no longer be a national Indigenous/non-Indigenous comparison. This was understandably very hard for the ABS to contemplate.

\section{Aims of the 2006 research project}

A primary purpose of observations in 2006 was to test the workability and impact of the reforms discussed above. In addition, the opportunity was presented for the first time to assess the nature and effectiveness of pre-census preparations and post-census processing. Two of the locality studies revisit places observed in 2001, while two others cover places being observed for the first time. The monograph also contains three chapters that observe related administrative processes leading up to and following on from enumeration in localities; preparing and undertaking training for the enumeration in the Darwin Census Management Unit (CMU), checking forms in the Darwin CMU after the field enumeration and, finally, the processing of these forms at the Data Processing Centre (DPC) in Melbourne (see also Morphy 2007). Our study this time has therefore been widened to include observation of 'back-office' administrative processes before and after the event, as well as enumeration in the field.

During the course of the research our focus shifted - as a result of what we were observing - to the broader structures and processes of the IES. The question of who was being counted where loomed ever larger as we watched the struggles of the Census Field Officers (CFOs) and their inadequate numbers of recruited field staff to maintain control of the process, and an orderly count, in the context of a prolonged engagement with a highly mobile population. We came to see that the strategy as presently conceived is ill-equipped to deal with the agency of a population for whom the census is essentially an unfathomable state project. It does not engage adequately with the resources and local knowledge embodied by the organisations that straddle the interface between these populations and the state. Nor is it designed to cope realistically with the contingencies that arise in everyday life in these remote communities.

\section{The Indigenous Enumeration Strategy in 2006: structures and processes}

The case-study chapters (Chapters 3-6) describe what really happened on the ground during the count. In this section, we outline briefly the structures and 
processes of the count as envisaged and planned for by the ABS for the Northern Territory IES. ${ }^{2}$

The IES was extended in time well beyond the process of enumeration itself-before and after-beginning in November 2005 with training for State Indigenous Managers (SIMs). SIMs were employed and CMUs established within ABS regional offices in each State and Territory. They were responsible for coordination of ensuing field operations associated with the Community Housing and Infrastructure Needs Survey (CHINS) and the census, including the training of the CFOs, who were responsible for organising the collection of CHINS and then census data in the regions to which they were assigned.

As in previous years, the IES involved firstly the designation of the communities and areas in which the remote strategy was to be applied. These then became the responsibility of the CFOs, whereas the 'mainstream' count was the responsibility of the local area supervisors. The IES involved the use of a form - the IHF - which differed in its structure and in the content of some of its questions from the form used in the mainstream count. In particular, the IHF was designed to attempt to capture data on household structure, education, employment and socio-cultural factors such as religion, language use and ethnicity, using questions and options for responses framed to allow for the 'difference' of remote Indigenous populations.

As in the past, the IES attempted also to mitigate the effects of remoteness and low levels of literacy in English in the remote Indigenous population. The census forms were not dropped off at households; rather, local CIs, ideally managed by local CCs, took the IHFs to the individual households in the communities and filled them in with the help of the household members. The responsibility for training these local temporary staff rested with the CFO for the region.

In the Northern Territory, it was decided - as in past censuses - that such a process made it logistically impossible for the count to take place on a single night. Accordingly - as in the past - the IES in the Northern Territory employed a rolling count over an extended period. The time frame initially allowed for the count was six weeks, and it was the responsibility of the CFO to organise the count within this time frame in their designated region. In 2006, for the first time, in acknowledgement that in reality this was a very exacting task, the CFOs were assigned an Assistant - also trained at the regional office - to go with them into the field. In the Northern Territory, a couple of 'floating' CFOs were trained, who were not assigned particular regions; their job was to provide backup wherever and whenever it was deemed necessary. In Western Australia, it was

\footnotetext{
2 The processes planned for Western Australia differed in some respects. These differences are discussed in the Fitzroy Crossing case study (Thorburn, Chapter 6).
} 
felt that the IES could be completed in a week, being in effect a slightly extended version of the more usual census method of counting on a single night.

In the best-case scenario, as detailed and emphasised in the training delivered to the CFOs, the IES should have proceeded as follows. During the initial visit to each community during the CHINS exercise, the CFO would also complete a form for each community, to be entered onto a new Discrete Indigenous Communities Database (DICD), which was being compiled for the first time for the National Centre for Aboriginal and Torres Strait Islander Statistics (NCATSIS). These data and the initial population figures revealed by the CHINS - together with the population figures from the 2001 Census for the community-would help the CFO to determine how many CCs and CIs would be needed to achieve a timely and complete count in the area. During this initial visit, the CFO would also carry out an awareness-raising exercise about the impending census by liaising with local community organisations and potentially helpful individuals, such as the census volunteers who were being recruited locally (for the first time) to provide logistical backup and assistance. If they had time, they might visit the local school and so on, and they had posters and other publicity materials to distribute in the communities. The CFO would also begin the process - with the help of local organisations - of identifying and meeting potential CCs for the census process.

After returning to the State or Territory CMU for a week's training on the IES procedures, the CFO with their Assistant would then return to their designated region to begin work. Their task, in each of the communities in their region, would be to recruit and train the necessary CCs and CIs for each community, sign them up as temporary ABS employees and ensure that all the details necessary for their payment were relayed to the CMU.

The first task after training would be for the CFO and the CC (or CCs) to go around the community - which had been assigned the unique identifier code for its Collection District (CD) - and compile a Master Dwelling Checklist (MDC) that would include all private dwellings, temporary dwellings and non-private dwellings in the community. Each separate dwelling would be assigned a unique Census Record Number (CRN). Non-Indigenous households and non-private dwellings would be identified and special arrangements made for the delivery and collection of the forms relating to them. Indigenous households, once identified, would be divided among the available CIs, using the local knowledge of the CCs to distribute the workloads most appropriately. Each CI would then be given an Interviewer Dwelling Checklist (IDC), on which the CD and the CRNs of each dwelling to be visited by them was to be listed, along with the surname of the 'head' of the household. They were to complete the details on this checklist as they went along. Once a household had been counted, the dwelling would 
be ticked off the list and the numbers of males and females in the household recorded on the IDC.

The task of the CCs - aided initially by the CFO until they were confident that the CCs had fully understood their responsibilities - would be to manage the CIs by ensuring that they visited all the dwellings on their list, and reassigning workloads if unplanned-for contingencies arose. It would be their task to collect and store securely the completed forms and to transfer the details of people counted from the IDCs to the MDC, having first checked that the forms had been completed fully and that the numbers of people on the IHFs tallied with the totals listed on the IDC. One copy of the IDC would be kept by the CI as a record of the pay owing to them, one would stay with the CC and one would be forwarded to the CMU by the CFO to initiate the payment process. It was envisaged - and it would be necessary if the count for the CFO's region was to be completed in the time allowed - that a day or two after the beginning of the count, the CFO could leave the community to start the same process in another of the CDs in their region, leaving the Assistant CFO behind for a while if this was felt to be necessary.

After the count was completed in a CD, and all the IHFs were returned to the CCs, the CFO would return to the community and double-check with the help of the CCs that all dwellings had indeed been visited and accounted for, that the totals of males and females on the MDC and the IDCs tallied and that all forms had been completed correctly. The CFO would also use available administrative data and the figures from the CHINS exercise and the 2001 Census to determine whether or not the coverage was complete. They would be alert for large discrepancies in totals, and for under-counting of particular sectors of the population, such as young men, children and infants. If there were any such discrepancies, they would be documented, followed up in the field and accounted for.

The CFO would then return all the forms for a CD, boxed together, to the CMU. There, further checks would be made (see Chapter 7) before the forms were finally sent en masse to the DPC in Melbourne. The boxes containing the IHFs would contain - in addition to the forms themselves - copies of the MDC and the IDCs, the completed DICD forms and two checklists, one completed by the CFO before the forms left the CD and one completed at the CMU.

This very quick sketch of what is a very complicated administrative and logistical exercise is designed as a necessary background to the case-study chapters. It will become obvious that the reality fell far short of the ideal, in the face of the complex realities encountered by the CFOs and their teams on the ground.

One final important step in the process - which was new in 2006- was the extension of the Post Enumeration Survey (PES), designed to estimate census net under-count (ABS 2007) in remote areas and discrete Indigenous communities 
covered by the IES. This survey is conducted by the ABS a month after, but independent of, the census, and is used to estimate numbers of people missed or counted more than once in the census enumeration and then to adjust population estimates accordingly. In the past, the PES has not been carried out in remote Australia and discrete Indigenous communities and the rate of people missed or counted twice there has been estimated as roughly equivalent to elsewhere. This survey was not observed by the current research team because of its independence as a process from the census itself. John Taylor (2007b: 18) reports that, according to the ABS's data, 'In Western Australia around 24 per cent of the Indigenous population was estimated to have been overlooked by the census; in the Northern Territory the figure was 19 per cent.' This recognition of a larger than normal under-count has significant implications for the use of census statistics in matters concerning Indigenous affairs funding. Our observations also show that the data collected for many of the people who were counted were incomplete (see Chapter 7). Together, these two facts point to some very serious problems with the IES.

We conclude that, despite the best intentions, the census is failing to capture adequately the characteristics of remote Indigenous populations. In Chapter 9, we call for a substantial rethink of the way in which the ABS engages with Indigenous communities and their organisations. 